This paper is the author accepted manuscript. The manuscript is in press at the journal Social Psychiatry and Psychiatric Epidemiology, Sept 2018

\title{
Development and predictors of mental ill-health and wellbeing from childhood to adolescence
}

Praveetha Patalay ${ }^{1,2}$, ORCID: 0000-0002-5341-3461

Emla Fitzsimons², ORCID: 0000-0003-4466-5963

1. Institute of Psychology, Health and Society, University of Liverpool

2. Centre for Longitudinal Studies, UCL Institute of Education, University College London, UK

Correspondence to:

Praveetha Patalay, University College London,

Gower Street,

London WC1E 6BT

UK

Email: p.patalay@ucl.ac.uk

Tel: +44 (0)2076126051

\section{Acknowledgements}

We are grateful for the co-operation of the Millennium Cohort Study families who voluntarily participate in the study. We thank the Economic and Social Research Council and the co-funding by a consortium of UK government departments for funding the Millennium Cohort Study through the Centre for Longitudinal Studies (CLS) at the UCL Institute of Education, London. We would also like to thank a large number of stakeholders from academic, policy-maker and funder communities and colleagues at CLS involved in data collection and management. The funders of the study had no role in study design, data collection, data analysis, data interpretation, or writing of this paper. 


\begin{abstract}
Purpose: Investigate the (1) longitudinal changes and sex differences in mental ill-health and wellbeing from ages 11 to 14, (2) predictors of changes in mental health outcomes, and (3) sex and reporter differences in these predictors.

Method: Data from 9553 participants in the Millennium Cohort Study, with both mental illhealth (parent- and self-report) and wellbeing outcomes of the cohort members measured at ages 11 and 14. A range of childhood socio-demographic, human capital, family and wider environment risk and protective factors are investigated.

Results: Wellbeing has weak stability and mental ill-health has moderate stability between ages 11 and 14 and large sex differences emerge in all the mental health outcomes investigated, with girls experiencing lower wellbeing and greater symptoms of mental illness at age 14. Raw associations between outcomes, and differences in their predictors, indicate varying patterns emerging for parent- and self-reported mental ill-health, with parent-reported symptoms in childhood a poor predictor of both self-reported wellbeing and depressive symptoms in adolescence. Investigating the emergent sex differences in prevalences highlights childhood risk and protective factors at this age that are more salient in females, including family income, school connectedness, cognitive ability, whereas peer relationships and bullying were equally relevant for mental health development in both males and females.

Conclusion: Low-moderate stability of mental health outcomes stresses the importance of the transition period for mental health, highlighting an intervention window at these ages for prevention. Socio-economic status is associated with mental health development in females but not in males at this age, highlighting a sex-specific vulnerability of deprivation associated with poorer mental health in adolescent females.
\end{abstract}

Keywords: wellbeing, transition, mental illness, depression, longitudinal 


\section{Introduction}

The burden of mental ill-health is increasing [1] and longitudinal research demonstrates that symptoms are first experienced in childhood and that for individuals who will experience psychiatric disorder though their lifetime, around half experience clinical levels of symptoms by the age of 14 years [2]. In comparison, relatively less is known about the stability and continuity of wellbeing through the lifecourse and the childhood predictors of subjective wellbeing. Although some consider wellbeing and mental ill-health to be two ends of the same spectrum of mental health, there is increasing evidence of their relatively weak correlations and the identification of a substantial number of individuals who experience good wellbeing in the presence of symptoms of mental ill-health [3-5]. This supports a two-domain view of mental health where mental ill-health and subjective wellbeing are distinct constructs, that are associated but only moderately $[4,5]$.

In beginning to investigate the childhood correlates of both mental ill-health and wellbeing, in Patalay \& Fitzsimons [5], we investigated a wide framework of predictors of both mental illhealth and wellbeing at age 11 years in a large, nationally representative sample in the Millennium Cohort Study (MCS). We discovered that at this age, parent-reported mental illhealth and self-reported subjective wellbeing, were weakly correlated. We also report that they had some common correlates across these two domains, but more unique predictors of mental ill-health and wellbeing emerged [5]. However, the lack of symptoms of mental ill-health as reported by the young individuals themselves was a major limitation of this work, clouding distinctions between these constructs and possible differences based on different reporter's perspectives. In addition, the outcomes in this work were cross-sectional and assessed at age 11. Longitudinal investigations of child wellbeing are scarce, with little known about the stability of wellbeing over time [6], especially during the childhood to adolescence transition, which is identified as a key transition for young people and corresponds to social and biological changes [7]. The extant longitudinal studies of children's wellbeing have focussed on how longitudinal changes in family income or parental employment affect cross-sectional wellbeing at a certain age [6], with few studies investigating changes in children's wellbeing [8]. Moreover, little is known about the longitudinal associations between these two domains.

The childhood-adolescent transition period is considered a pivotal period for prevention, with many physical health and risky health behaviour prevention programmes targeting this developmental period [9], whereas for mental health this is less the case [10]. The current limited 
understanding of the influences of transitionary periods on mental health thereby also limits the capacity to design and implement effective interventions for this age group. Hence, this paper aims to extend the research literature investigating child mental ill-health and wellbeing [5] in three significant ways:

1) Longitudinal change: Investigate the developmental stability and changes in individuals' mental health (both ill-health and wellbeing) between ages 11 and 14 years, a period in which many key transitions are experienced, including the transition between primary and secondary school, and puberty. Examine how mental ill-health at age 11 predicts changes in wellbeing between 11 at 14, and how wellbeing at 11 predicts changes in mental ill-health between 11 and 14.

2) Reporter effects: Examine whether mental ill-health and subjective wellbeing are more closely associated and their predictors more closely aligned when the reporter of mental ill-health is the same. This is facilitated by the availability, for the first time in this cohort, of parent- and selfreported mental ill-health symptoms.

3) Sex differences: Investigate if there are predictors of changes in symptoms from ages 11-14 that vary by sex, to identify predictors that help explain the sex difference in prevalence that emerges between ages 11 and 14. This is motivated by the large differences in prevalence of psychological distress that emerge between these two ages [11-14]. For instance, in this cohort, $12 \%$ of boys and girls at age 11 years have significant emotional problems as reported by parents, which remains the same in boys (12\%) but increases to $18 \%$ in girls at age 14 [11]. Self-reported depressive symptoms indicate that $9 \%$ of boys and $24 \%$ of girls at age 14 have high depressive symptoms [11].

In summary, the current study aims to 1) establish the associations between mental ill-health, as reported by both parents and young people, and subjective wellbeing at age 14 years, and examine longitudinal stability in these outcomes from ages 11 to 14 years, in a large, nationally representative cohort study, 2) investigate the childhood predictors of mental ill-health and mental wellbeing outcomes at age 14 and their longitudinal change from ages 11 to 14 , and 3) examine sex differences in associations, stability and predictors of change in mental health from ages 11 to 14 years. 


\section{Method}

\section{Participants}

Participants are from the Millennium Cohort Study, a UK-wide birth cohort study of just over 19,500 individuals born at the start of the millennium (Sept. 2000 - Jan. 2002). Greater details on the study design, variables and response can be found at: www.cls.ioe.ac.uk [15]. In the current study 9553 cohort members who had available the outcome measures of interest (mental ill-health, depressive symptoms and subjective wellbeing) at ages 11 and 14 years are included in the analysed sample. The analysed sample is $50.85 \%$ female, and $84.4 \%$ identified as White, 8.7\% Asian, 2.8\% Black, 2.7\% mixed ethnicity and 1.4\% as other ethnicities. Ethics permissions for each wave of data collection were received as described in the study documentation [15].

Of the 19243 potentially eligible families at the age 14 sweep, 15415 were issued into the field (those not issued due to emigration, permanent refusal, untraceability) of which 11726 families participated in the age 14 sweep (overall response rate of 60.9\%)[16]. The analysed sample included the subsample who had both parent- and self-reported assessments of the key mental health outcomes at both key sweeps (ages 11 and 14) that are the focus of this study. The age 14 sample was compared to the full MCS sample on key demographic criteria. Participants were more likely to be missing if the cohort member was male, Black ethnicity, lower occupational and educational level of the parents and single parent family [17]. Sample and attrition weights created for the study are used in all analyses [16].

\section{Measures}

Overall, this paper focus on five mental health outcome measurements spanning two sweeps (ages 11 and 14) of the study. At age 11, mental ill-health of the cohort member was reported by their parents and wellbeing was reported by the cohort member themselves. In the recent age 14 assessments of the MCS members, internalising mental ill-health represented by emotional/depressive symptom measures was reported both by the parent and the cohort member. In addition, subjective wellbeing was reported by the cohort member using the same measure as at age 11 . 
Mental ill-health (parent-report; ages 11, 14)

Symptoms of mental ill-health difficulties experienced by the cohort member, representing the two broad domains of prevalent symptomatology in childhood - internalising and externalising problems - were measured using the emotional symptoms and conduct problems subscales of the Strengths and Difficulties Questionnaire [18], a widely used questionnaire of symptoms of mental illness in children in the UK since the late 1990s. A parent/carer ( 95\% mothers) responded by indicating how true (not true, somewhat true, certainly true) statements about the cohort member are. In this study we also aggregate the items of both scales to create a mental ill-health score, with higher scores indicating greater symptoms, and this is controlled for at age 11 in the analyses of predictors of age 14 symptoms [19]. In this dataset the estimated internal consistencies of the scales are: emotional symptoms scale (age $11 \alpha=.72$, age $14 \alpha=.72$ ) and conduct problems scale (age $11 \alpha=.62$, age $14 \alpha=.59$ ). At age 14 , we focus on the predictors of the emotional symptoms subscale as it measures symptomology that is most similar to the age 14 self-report depressive symptom measure described below.

\section{Depressive symptoms (self-report, age 14)}

Cohort members completed the Short Moods and Feelings Questionnaire which assesses symptoms of depression in young people [20]. The measure consists of 13 items that assess feelings or behaviours in the previous fortnight (e.g. I felt miserable or unhappy) with responses on a 3-point scale: True, Sometimes, Not true. The items are aggregated to create a depressive symptoms score, with higher scores indicating greater symptoms. Internal consistency is high ( $\alpha$ $=0.93)$.

\section{Mental wellbeing (self-report, ages 11, 14)}

Mental wellbeing was assessed using a six-item measure assessing satisfaction with different aspects domains which are appropriate to children including school, family, friends, school work, appearance, and life as a whole [21]. Cohort members responded by indicating their level of happiness with each aspect of their lives on a 7-point scale ranging from not at all happy to extremely happy. The score is aggregated to represent overall wellbeing, with higher scores indicating greater wellbeing. The internal consistency of this measure is high (age $11 \alpha=.83$, age $14 \alpha=.86)$. 


\section{Predictors}

In previous research [5], we examined a large, comprehensive list of correlates of mental illhealth and wellbeing at age 11 years. In this study we have selected the sub-group of variables that were significantly associated with either wellbeing or mental ill-health at age 11, in order to examine their association with change in these outcomes from ages 11 to 14 . We examine predictors assessed in childhood (most at 11 years, further details in [5]) in 4 key areas, from the individual level to their family, social, school and wider environment - 1) socio-demographic factors which include demographic characteristics, socio-economic status and perceived wealth and inequality, 2) human capital factors including cognitive abilities and health status, 3) family factors including family structure, home environment and parent health, 4) social and environmental factors including social relationships and aspects of the wider school and neighbourhood environments.

\section{Socio-demographic factors}

Cohort member demographic characteristics included were gender (coded $0=$ male, $1=$ female) and ethnicity grouped into 5 broad groupings of White, Asian, Black, mixed and other ethnic groups. Household income was represented in UK equivalised quintiles ( $1=$ lowest income quintile, $5=$ highest income quintile). We also include cohort members' perceptions of SES. Cohort members' satisfaction with their family's wealth was measured using an adaptation of Schor's consumer involvement scale [22], which assesses how satisfied they are with their family's material possessions and wealth (e.g. I wish my family could afford to buy me more of what I want), higher scores indicating greater dissatisfaction. Their perception of relative wealth/inequality was assessed with their responses to the question, "Compared to your friends, is your family richer, poorer or the same?"

\section{Human capital factors}

Cohort member's cognitive ability was assessed using a latent factor score derived from the British Ability Scales [23], using the naming vocabulary, picture similarity, pattern construction and verbal similarities scores at ages 3, 5, 7 and 11 years respectively. Learning and communication difficulties were assessed by whether the cohort member has a special educational needs status and parent reports of communication difficulties. Physical health markers included in analysis are presence of chronic illness, based on indication from a parent on whether the cohort member suffers from any serious or chronic illness (e.g. asthma, diabetes), 
and overweight status based on whether the cohort member's measured BMI exceeded the overweight threshold based on the classification of the International Obesity Task Force [24].

\section{Family factors}

The characteristics of the family that were included in analyses are whether the cohort member lives in a single parent household (=1) and number of siblings $(0,1,2$ or $3+)$. All of these data are available from the household grid in the MCS. Variables related to the home environment that are included in analysis include an indication by parents of frequently arguing with the cohort member ( $1=$ at least once a week), and cohort member reports of whether they were frequently bullied by their siblings ( $1=$ bullied by siblings at least once a week). Parent health and well-being variables are aggregated to the household level and include parent mental health measured using the Kessler K6 scale [25], general health measured with the general health item from the SF-8 ("how would you describe your health generally?") and life satisfaction measured using a single item measure of life satisfaction "On a scale from 0 to 10 how satisfied are you about the way your life has turned out so far?", where higher scores indicate greater life satisfaction.

\section{Social and environmental factors}

Both cohort member and parent perspectives of the cohort member's social relationships were included. Parents reported on problems in the cohort member's relationship with peers with the peer problems SDQ subscale [18]. Cohort member's reports on their relationships with friends were assessed by asking them how often they argued or fell out with their friends $(1=$ at least once a week/have no friends). Cohort members also reported on whether and how often they were bullied by their peers ( $1=$ bullied at least once a week). Cohort member's perspectives on their feelings of connectedness with and interest in their school and whether they liked school were included to capture their assessment of their school environment and the belonging-ness and interest they felt in school. Perceived neighbourhood safety was assessed in response to the question "How safe is it to walk, play or hang out in this area during the day?"(very safe to not at all safe; $1=$ not very safe/not at all safe). Lastly, country of domicile within the UK - England, Scotland, Wales or Northern Ireland - was included in analysis. 


\section{Analysis}

In the selected analysis sample of 9553 no data were missing for the outcome variables and socio-demographic characteristics (sex, ethnicity, income). Multiple imputations were carried out to impute values on missing predictors. Overall missing cells were at less than $2 \%$ of the total, and 10 imputed datasets were created for analysis. Given the stratified clustered sample design of the MCS and to account for sub-group oversampling and attrition over waves, all analyses were conducted accounting for the survey design and applying weights $[16,26]$.

First, we report the mean scores of each mental health outcome measure at both ages and examine correlations between these variables. We also present sex differences in these scores, estimate correlations between the mental health variables and test whether there are any sex differences in the strength of the correlations using a Fisher's $\mathrm{Z}$ transformation approach to investigate between group differences in correlations [27]. To examine the correlates of the mental health outcomes at age 14 years, regression analyses were conducted predicting standardised z-scores of the outcome variables, permitting the comparison of coefficients across models. We then examine these outcomes conditioning on both mental ill-health and wellbeing at age 11 years, hence focussing on the predictors of mental health development or change between ages 11 and 14 years. Lastly, we examine interactions with sex to estimate whether the predictors vary in males and females. Based on improvement in model fit when including interactions with sex, we present these models predicting changes in the outcomes separately for males and females, indicating which predictors are moderated by sex and which are not.

\section{Results}

\section{The relationship between mental ill-health and wellbeing}

The correlation between parent-reported mental ill-health and self-reported wellbeing is weak, both at ages $11(-0.18)$ and $14(-0.25)$. On the other hand, self-rated mental ill-health is associated moderately with wellbeing at 14 years (-0.58), indicating much higher proportion of common variance between these two domains when assessed by the young person themselves at this age (this comparison is not possible at age 11 given the absence of a self-reported mental ill-health measure at 11).

From age 11 to 14 years overall changes in mean scores of mental ill-health and wellbeing demonstrate an increase in parent-reported mental ill-health and a decrease in overall self- 
reported wellbeing. The stability estimates indicate higher stability in parent-reported mental ill-health (0.61), compared to subjective wellbeing (0.25). Stability in self-reported symptoms cannot be assessed given the lack of a self-reported measure of mental ill-health at age 11 .

In terms of sex differences, differences in average standardised scores in the two samples can be seen in Figure 1. At age 11 years there are no significant sex difference in wellbeing or the broad mental ill-health construct; when looking separately at emotional and conduct scales, we see that females on average have higher emotional and lower conduct problem scores compared to males. On the other hand, at age 14 years, there are stark sex differences across all (except conduct problems) outcomes, whereby females have significantly higher internalising symptoms as reported both by themselves and their parents, and have significantly lower wellbeing. The sex difference in conduct problems is no longer observed suggesting that the difference observed in overall parent-reported mental ill-health is driven by sex differences in emotional problems. The correlations by sex are similar in some instances (see Table 1; e.g. between emotional and conduct problems at both ages, wellbeing and conduct problems, self-reported depressive symptoms and parent reported conduct problems at age 14). The correlations between mental illhealth at 11 and age 14 outcomes suggested stronger associations with age 14 wellbeing in females, but stronger association with parent-reported conduct problems in males. The correlations between mental ill-health and wellbeing at age 14 were significantly different by sex with stronger associations between mental ill-health and wellbeing in females (e.g. selfreported depressive and parent-reported emotional symptoms and wellbeing were -.63 and -.25 in females compared to -.47 and -.14 in males).

\section{Predictors of mental health at age 14}

In Table 2, we observe the childhood predictors of the three mental health outcomes at age 14 years. At this age, females experienced significantly greater symptoms and poorer wellbeing. Lower family income, arguing with parents, poorer parent health and low school connectedness were associated with poorer mental health across the three outcomes at age 14 .

The following factors were associated with some but not all outcomes at age 14. Asians reported better self-reported mental health outcomes. Higher perceived wealth (relative to friends), being overweight or obese, being bullied by peers all predicted poorer self-reported mental health (i.e. lower wellbeing, greater depressive symptoms). Higher cognitive ability predicted lower parentreported symptoms, but greater self-reported symptoms and poorer wellbeing. Cohort members with longstanding chronic illness had higher symptoms as reported by their parents, but not 
themselves, and lower self-reported wellbeing. Parent mental health showed a stronger association with parent-reported symptoms than with self-reported mental health. In terms of country of domicile, 14-year-olds in Northern Ireland had significantly better wellbeing and lower depressive symptoms than their peers in other parts of the UK.

\section{Predictors of mental health at age 14, conditional on mental health at age 11}

As can be seen in Table 2, many of the predictors of the change from age 11 to 14 years were similar to the predictors of mental health at age 14 years, especially for wellbeing where there is low longitudinal stability in scores. Wellbeing at age 11 was a better predictor than parentreported mental ill-health at age 11 of depressive symptoms at age 14 and parent-reported mental ill-health at 11 did not predict changes in wellbeing scores from 11-14.

Being female and having peer problems at age 11 predicted poorer mental health, irrespective of reporter at age 14, when controlling for mental health at age 11. In terms of self-reported mental health outcomes at 14, lower family income, parent general health, peer problems, being bullied by peers and cognitive ability were associated with worse self-reported mental health between 11 and 14 years. Poor parent mental health did not predict poorer self-reported mental health.

Better mental health outcomes at 14 were mainly predicted by wider social factors such as school connectedness, liking school and living in Northern Ireland.

\section{Sex differences in predictors of mental health at age 14}

As shown in Figure 1, and reported elsewhere in greater detail [11], a gender gap appears between ages 11 to 14 years, whereby the levels of symptoms being experienced by girls sharply increases between these ages. For each outcome, a model with sex interactions was examined and the specific predictors for which there was a sex difference in the predictors association with outcome, are indicated in bold in Table 3. We report the predictors of symptom development from age 11 to 14 separately by sex (Table 3), to ascertain whether there are some risk and protective factors unique to females that might begin to start explaining the emergence of this gender disparity in mental ill-health. Previous mental ill-health (parent-reported) only predicted parent-reported mental ill-health at age 14 in both boys and girls (larger effect in girls) and poorer wellbeing in girls at 14 . Wellbeing at 11 was a significant predictor of both depressive symptoms and wellbeing at 14 in both males and females. 
Asian and Black females seem to experience less worsening of mental health between these ages than White females, and a similar pattern was observed in both boys and girls with regards to wellbeing. The relationship between income and symptoms at age 14 is noteworthy, with no income differences in depressive symptoms or wellbeing observed among boys, whereas girls from lower income families were experiencing greater depressive symptoms and poorer wellbeing at age 14 years, with evidence of a gradient in severity based on different levels of family income. Low material position of family, higher cognitive ability, special educational needs and lower school connectedness were linked to poorer self-reported mental health in females and not in males. Girls living in Northern Ireland had significantly better mental health, an association not observed in boys.

\section{Discussion}

This study presents data on the stability and changes in mental health, both ill-health and wellbeing across the childhood-adolescence transition, a period characterised by the move from primary to secondary schooling, parent to peer centric socialisation and the experience of physiological changes related to puberty. We also examine the childhood predictors of longitudinal changes in outcomes between these ages, focussing on understanding the childhood predictors of emerging sex differences in prevalence and how the results vary when mental illhealth is reported by the parent or the young person themselves.

Prevalence data at age 11 indicate no significant sex differences in wellbeing and overall mental ill-health, which when broken down into emotional and conduct suggests higher emotional problems in girls and higher conduct problems in boys; this is similar to findings from other research in this age group $[19,28]$. At age 14, however, overall mental ill-health is higher in girls which is driven by even higher levels of emotional problems at this age relative to boys. As expected from the literature on this topic, this clear sex difference is also observed for selfreported depressive symptoms at this age [14], with girls in this study, on average, scoring half a standard deviation higher on depressive symptoms. A less established finding relates to sex differences in wellbeing, where 14-year old girls scored one-third of a standard deviation lower on average.

Wellbeing between ages 11 and 14 was far less stable compared to parent-reported mental illhealth. The moderate stability in psychopathology is in line with other research in this field [29, 30], whereas findings on sex differences in stability of psychopathology are inconsistent in the literature. The poor stability in wellbeing is a novel finding of this study as little has been 
published regarding the stability of wellbeing across childhood and adolescence, whereas in comparison wellbeing in adulthood has been found to be more stable [31]. In terms of changes in the outcomes, wellbeing at age 11 was a better predictor of depressive symptoms at age 14 compared to parent-reported mental ill-health at age 11. Moreover parent-reported symptoms did not predict any of the changes in wellbeing scores from 11-14. This is a striking finding which highlights not only that the chasm between self-reports and parent-reports of symptoms are cross-sectionally large, but also that parent perspective of their child's mental health difficulties is a weak predictor of their later mental health outcomes as well.

The predictors of changing mental health over time include relatively low family income, dissatisfaction with family material position, special educational needs, being bullied by peers or siblings, being overweight - all predicting worsening mental health outcomes between 11 and 14 years. Higher cognitive ability predicted worse self-reported mental health outcomes and better parent-reported mental ill-health at age 14; this finding requires further detailed study. Stark sex differences emerge in both mental health and wellbeing between 11 and 14, whereby adolescent girls are more likely to have poorer scores in both ill-health and wellbeing, irrespective of reporter. This contrasts with research evidence from adults, where females are more likely to experience higher symptoms of mental ill-health, but also greater wellbeing [32, 33]. The associations between these different domains of mental health, however, were broadly similar in males and females, with the exception of wellbeing and depressive symptoms at age 14 where the association between these two variables was stronger in females than in males. The emergence of these stark sex differences in severity and prevalence prompted investigating the predictors these outcomes separately by sex.

Experience of socio-economic deprivation, both contemporaneous and throughout childhood, have been found not to be associated with children's subjective wellbeing at age $11[5,6]$. By age 14 we see that this is no longer the case; low family income predicts lower wellbeing at age 14 and there is a clear income gradient in predicting wellbeing. However, when analysing this separately by sex, we see that this income differential in subjective wellbeing is observed in girls, with unclear relationships between family income and boys' wellbeing at 14 . This picture is mirrored in the depressive symptom outcomes at 14 whereby there is no association between family income and depression in boys, whereas a clear association emerges in girls. There are significant associations between parent-reported mental ill-health and income for both boys and girls, however, the associations are stronger and patterns clearer in girls than in boys. These findings strongly suggest that the observed socio-economic gradient observed in adult mental 
health outcomes [34, 35] first appears between the ages of 11 and 14 in girls and will possibly appear in boys at a later stage of adolescence. The sex differences observed might reflect differences in age at pubertal onset and developmental sex differences at these ages. The observing of inequalities in mental health at a later age is in contrast to other cognitive and health domains such as cognitive scores and obesity, where socio-economic differentials are evident from earlier in childhood $[36,37]$. This finding highlights the need to understand the drivers of the emergence of these socio-economic inequalities at this age, which will also inform prevention efforts for mental health and policy efforts to reduce inequalities in health outcomes [38].

This paper also aimed to disentangle the effects the reporter of mental ill-health has on understanding the relationship between mental ill-health and wellbeing. It is common practice in child and adolescent mental health research for the parent to be the reporter of the young person's mental health, despite decades of evidence highlighting low agreement between these reporters $[39,40]$. The findings at age 14 suggest that mental ill-health and wellbeing have a much stronger correlation when reported by the young person compared to when reported by the parent. However, the correlation even within the same reporter (young person) was moderate, with some variation in predictors of these two outcomes, for instance, chronic illness, number of siblings and parent life satisfaction significantly affected only self-reported wellbeing scores, and Black ethnicity and parent mental ill-health significantly affected only the self-reported depressive symptoms. These findings further highlight the unique insights that are gained when young people's own perspectives of their mental health are recognised in research and practice. Selfreport measures of mental health difficulties have been developed and validated for children as young as 7, age appropriate measures being key to asking about these difficulties from a younger age $[41-43]$.

The research reported in this paper is supported by the strength of this large, nationallyrepresentative, longitudinal cohort which has collected both wellbeing and mental ill-health data at different ages, making possible this first contribution of longitudinal changes in child wellbeing and its predictors to the literature. However, this preliminary investigation has focused on childhood predictors of changes in mental health scores across the childhood-adolescence transition; future research should extend this by examining contemporaneous factors in adolescence and how changes in social, biological and school transitions between these ages impact on changes in these domains between ages 11 and 14. By studying the effects of changes in predictors on changes in these outcomes the longitudinal nature of the data can be further 
exploited to understand how changing circumstances at these ages impacts on the development of children's mental health.

While there is an increased interest in studying wellbeing in recent years, children's wellbeing remains understudied in comparison with adult wellbeing (and compared to mental ill-health) $[5,44]$. This is the first study to investigate changes in wellbeing across the childhoodadolescence transition and compare the key predictors of longitudinal changes of mental illhealth and wellbeing at these ages. Understanding mental health development in childhood and adolescence is important as mental health in childhood is predictive of a range of adult outcomes $[45,46]$ and understanding the longitudinal developments in these domains at these life stages help understand the vast impacts these variables have through the lifecourse ultimately informing policy and prevention efforts in mental health. We find that both domains of wellbeing and mental ill-health in childhood are not strong predictors of the other domain a few years on, further lending support to the notion that wellbeing and mental ill-health are not just two ends of the same spectrum [47]; a perspective which to-date has been mainly supported by weak correlations in cross-sectional data $[3,4]$. The weak prediction of scores in one domain on future scores in the other domain, support arguments for measuring both wellbeing and mental ill-health as suitable as outcomes of interventions, especially when they focus on prevention and promotion $[48,49]$.

The findings on income inequality gradients emerging in wellbeing and depressive scores in 14 year old girls, but not boys, highlights two main things: 1) socio-economic gradients in selfreported mental health outcomes are observed later in the lifecourse compared to other health outcomes [50], and 2) the timing of when they appear is different for boys and girls, with it being evident in girls but not boys in early adolescence. Future research as the cohort gets older will help determine the age at which socio-economic gradient in mental health is evident for boys. The findings suggest that differences in access to resources, social support, and individual's choices through childhood and in early adolescence might begin to affect the emergence of socioeconomic differences in self-reported mental health with age [51].

The study has several strengths including the large national representative sample, and the availability of mental ill-health and wellbeing measures bridging key transitions such as the transition from primary to secondary school. However, limitations must be noted including the lack of a self-reported mental ill-health measure at age 11, the substantial levels of attrition in the cohort study, the availability of limited mental health measures in the study including the 
lack of a self-report measure that was comparable to the parent-reported conduct problems measure, and the use of the SDQ which covers limited aspects of mental illness and has moderate reliability.

In conclusion, the present study examines changes in mental ill-health and wellbeing between the ages of 11 and 14, highlighting the low stability in mental wellbeing and moderate stability of mental ill-health between these ages, providing evidence in support for the transitions at this age being a period that is suitable for intervention and prevention efforts [52]. In addition, the study shines a light on the different results that are obtained for parent and young person reported mental health, highlighting the poor predictive capacity of parent reported symptoms for future severity of outcomes for both depressive symptoms and wellbeing. The childhood predictors of changes in these domains are investigated with a focus on understanding the sex-differences that emerge in the severity of these outcomes between these ages. We discover that family income is only associated with mental health development in girls but not in boys at this age, highlighting a sex-specific vulnerability associated with early adolescent females with regard to their mental health.

Ethics statement. Research reported in this paper received ethical permission (details for ethics approvals for the different sweeps of the cohort are reported in the cohort documentation). The research was performed in accordance with the ethical standards laid down in the 1964 Declaration of Helsinki and its later amendments.

Conflicts of interest. The authors declare that they have no conflict of interest. 


\section{References}

1. Murray, C.J.L., et al., Disability-adjusted life years (DALYs) for 291 diseases and injuries in 21 regions, 1990-2010: a systematic analysis for the Global Burden of Disease Study 2010. The Lancet, 2012. 380(9859): p. 2197-2223.

2. NHS England and Department of Health, Future in mind: Promoting, protecting and improving our children and young people's mental health and well-being. 2015, NHS England \& Department of Health: London.

3. Sharpe, H., et al., Exploring the relationship between quality of life and mental health problems in children: implications for measurement and practice. European Child and Adolescent Psychiatry, 2016. 25(6): p. 659-667.

4. Kinderman, P., et al., Causal and mediating factors for anxiety, depression and well-being. $\mathrm{Br} \mathrm{J}$ Psychiatry, 2015. 206(6): p. 456-460.

5. Patalay, P. and E. Fitzsimons, Correlates of Mental Illness and Wellbeing in Children: Are They the Same? Results From the UK Millennium Cohort Study. Journal of the American Academy of Child \& Adolescent Psychiatry, 2016. 55(9): p. 771-783.

6. Rees, G., The Association of Childhood Factors with Children's Subjective Well-Being and Emotional and Behavioural Difficulties at 11 years old. Child Indicators Research, 2017.

7. The World Bank, World Development Report 2007: Development and the Next Generation. 2006, The International Bank for Reconstruction and Development / The World Bank: Washington D.C.

8. Robson, K., Changes in Family Structure and the Well-Being of British Children: Evidence from a Fifteen-Year Panel Study. Child Indicators Research, 2010. 3(1): p. 65-83.

9. Graber, J., J. Hill, and M. Saczawa, Childhood and the Entry into Adolescence: A Pivotal Period in Health-Related Behaviors and Prevention, in Defining Prevention Science, Z. Sloboda and $\mathrm{H}$. Petras, Editors. 2014, Springer US. p. 59-86.

10. Rice, F., et al., Identifying factors that predict successful and difficult transitions to secondary school. 2015, University College London: London.

11. Patalay, P. and E. Fitzsimons, Mental ill-health among children of the new century: trends across childhood with a focus on age 14. 2017, Centre for Longitudinal Studies: London.

12. Costello, E.J., W. Copeland, and A. Angold, Trends in psychopathology across the adolescent years: What changes when children become adolescents, and when adolescents become adults? Journal of Child Psychology and Psychiatry, 2011. 10: p. 1015-1025.

13. Patalay, P., H. Sharpe, and M. Wolpert, Internalising symptoms and body dissatisfaction: untangling temporal precedence using cross-lagged models in two cohorts. Journal of Child Psychology and Psychiatry, 2015. 56: p. 1223-1230.

14. Nolen-Hoeksema, S. and J.S. Girgus, The Emergence of Gender Differences in Depression During Adolescence. Psychological Bulletin, 1994. 115(3): p. 424-443.

15. Millennium Cohort Team, Millennium Cohort Study. A guide to the datasets (Eight Edition). 2014, London: Centre for Longitudinal Studies. 
16. Fitzsimons, E., Millennium Cohort Study Sixth Survey 2015-2016: User Guide (First Edition), E. Fitzsimons, Editor. 2017, Centre for Longitudinal Studies: London.

17. Mostafa, T. and G.B. Ploubidis, Millennium Cohort Study, Sixth Survey 2015-2016: Technical report on response (Age 14). 2017, Centre for Longitudinal Studies: London.

18. Goodman, R., The Strengths and Difficulties Questionnaire: A research note. J Child Psychol Psychiatry, 1997. 38: p. 581-86.

19. Patalay, P., et al., A general psychopathology factor in early adolescence. British Journal of Psychiatry, 2015. 207: p. 15-22.

20. Angold, A., et al., The development of a short questionnaire for use in epidemiological studies of depression in children and adolescents. International Journal of Methods in Psychiatric Research, 1995. 5: p. 237-49.

21. Taylor, M.F., et al., British Household Panel Survey User Manual Volume A: Introduction, Technical Report and Appendices. 2010, University of Essex: Colchester.

22. Schor, J., Born to buy: The commercialized child and the new consumer culture. 2004, New York: Simon and Schuster.

23. Elliott, C.D., P. Smith, and K. McCulloch, British Ability Scales second edition (BAS II): administration and scoring manual. 1996, London: Nelson.

24. Cole, T. and T. Lobstein, Extended international (IOTF) body mass index cut-offs for thinness, overweight and obesity. Pediatric obesity, 2012. 7: p. 284-294.

25. Kessler, R.C., et al., Screening for serious mental illness in the general population. Archives of general psychiatry, 2003. 60(2): p. 184-189.

26. StataCorp, Stata statistical software: Release 14. 2015, College Station, TX: StataCorp LP.

27. Kanji, K., Z-test for two correlation coefficients., in 100 statistical tests., K. Kanji, Editor. 2006, Sage: London. p. 42-43.

28. Patalay, P., et al., Cross-Domain Symptom Development Typologies and Their Antecedents: Results From the UK Millennium Cohort Study. Journal of the American Academy of Child \& Adolescent Psychiatry, 2017. 56(9): p. 765-776.

29. Deighton, J., et al., Longitudinal pathways between mental health difficulties and academic performance during middle childhood and early adolescence. British Journal of Developmental Psychology, 2018. 36(1): p. 110-126.

30. Tram, J.M. and D.A. Cole, A multimethod examination of the stability of depressive symptoms in childhood and adolescence. Journal of Abnormal Psychology, 2006. 115(4): p. 674.

31. Diener, E. and M. Eunkook Suh, Subjective well-being and age: An international analysis. Annual review of gerontology and geriatrics, 1997. 17: p. 304-324.

32. Graham, C. and S. Chattopadhyay, Gender and well-being around the world. International Journal of Happiness and Development, 2013. 1(2): p. 212-232. 
33. Piccinelli, M. and G. Wilkinson, Gender differences in depression. The British Journal of Psychiatry, 2000. 177(6): p. 486-492.

34. World Health Organization and Calouste Gulbenkian Foundation, Social determinants of mental health. 2014, World Health Organization: Geneva.

35. Allen, J., et al., Social determinants of mental health. International Review of Psychiatry, 2014. 26(4): p. 392-407.

36. Stamatakis, E., J. Wardle, and T.J. Cole, Childhood obesity and overweight prevalence trends in England: evidence for growing socioeconomic disparities. International journal of obesity, 2010. 34(1): p. 41-47.

37. Najman, J.M., et al., The generational transmission of socioeconomic inequalities in child cognitive development and emotional health. Social science \& medicine, 2004. 58(6): p. 11471158.

38. Siegrist, J. and M. Marmot, Social inequalities in health: new evidence and policy implications. 2006, Oxford: Oxford University Press.

39. Achenbach, T.M., S.H. McConaughy, and C.T. Howell, Child/adolescent behavioral and emotional problems: Implications of cross-informant correlations for situational specificity. Psychological Bulletin, 1987. 101: p. 213-232.

40. Rescorla, L.A., et al., Cross-informant agreement between parent-reported and adolescent selfreported problems in 25 societies. Journal of Clinical Child \& Adolescent Psychology, 2013. 42(2): p. 262-273.

41. Sharp, C., I.M. Goodyer, and T. Croudace, The Short Mood and Feelings Questionnaire (SMFQ): a unidimensional item response theory and categorical data factor analysis of self-report ratings from a community sample of 7-through 11-year-old children. J Abnorm Child Psychol, 2006. 34(3): p. 379-91.

42. Arseneault, L., et al., Psychometric evaluation of 5-and 7-year-old children's self-reports of conduct problems. J Abnorm Child Psychol, 2005. 33(5): p. 537-550.

43. Patalay, P., et al., Clinical validity of the Me and My School questionnaire: a self-report mental health measure for children and adolescents. BMC Child and Adolescent Psychiatry and Mental Health, 2014. 8: p. 17.

44. Holder, M.D., Happiness in children: Measurement, correlates and enhancement of positive subjective well-being. 2012, London: Springer

45. Copeland, W.E., et al., Adult functional outcomes of common childhood psychiatric problems: A prospective, longitudinal study. JAMA Psychiatry, 2015. 72(9): p. 892-899.

46. Layard, R., et al., What Predicts a Successful Life? A Life-course Model of Well-being. The Economic Journal, 2014. 124(580): p. F720-F738.

47. Westerhof, G.J. and C.L.M. Keyes, Mental Illness and Mental Health: The Two Continua Model Across the Lifespan. Journal of Adult Development, 2010. 17(2): p. 110-119.

48. Weare, K. and M. Nind, Mental health promotion and problem prevention in schools: what does the evidence say? Health promotion international, 2011. 26(suppl 1): p. i29-i69. 
49. Patalay, P., et al., Mental health provision in schools: approaches and interventions in 10 European countries. Global Mental Health, 2017. 4.

50. Bradley, R.H. and R.F. Corwyn, Socioeconomic status and child development. Annu Rev Psychol, 2002. 53(1): p. 371-399.

51. Li, J., et al., Social determinants of child health and well-being. Health Sociology Review, 2009. 18(1): p. 3-11.

52. Evangelou, M., et al., What makes a successful transition from primary to secondary school? 2008, Department for Children, Schools \& Families: London. 
Fig. 1 Mean standardised scores (with 95\% CIs) for the mental health variables at ages 11 and 14 for males and females.

\section{$\mathrm{PR}=$ parent-report and $\mathrm{SR}=$ self-report}

\section{Sex differences in mental health outcomes at age 11 and 14}

- Males Females

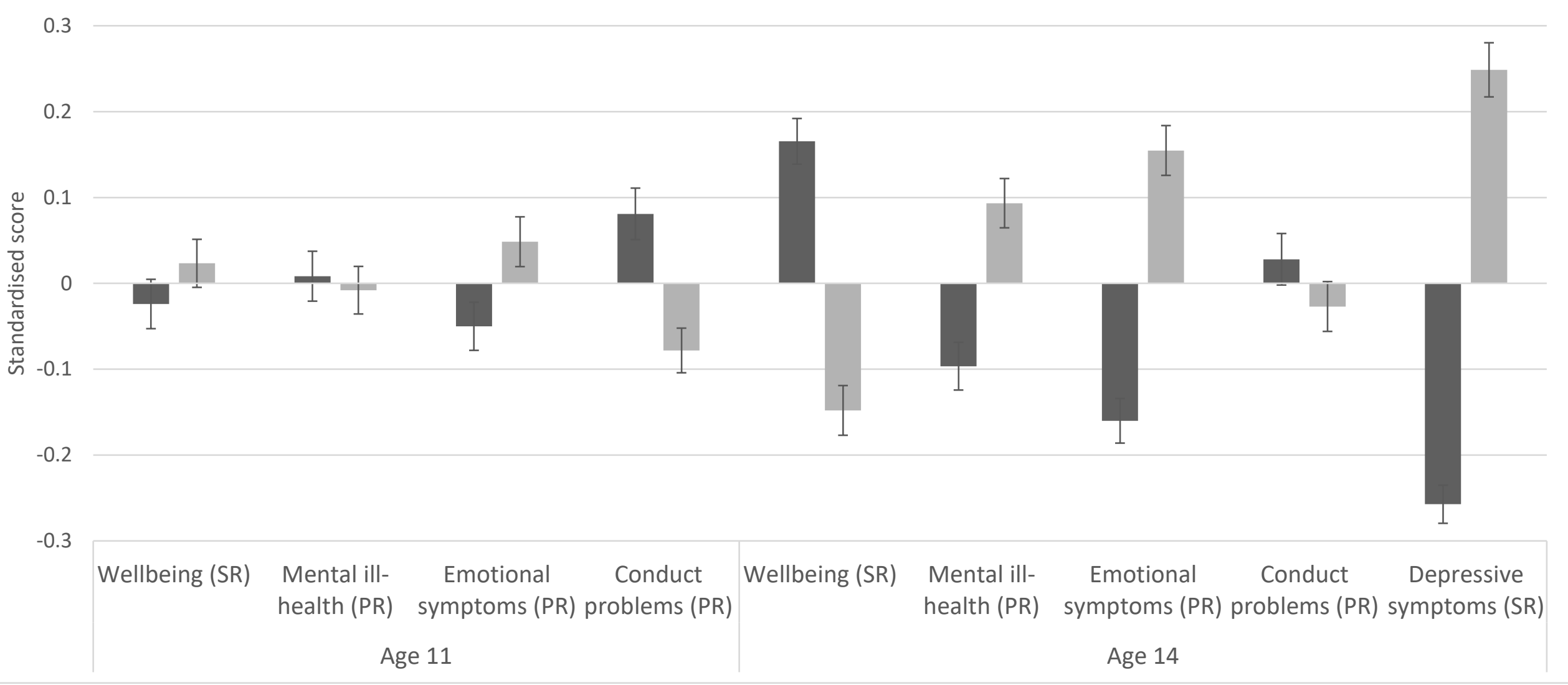


TABLES

Table 1 Correlations of mental ill-health and wellbeing at ages 11 and 14 years

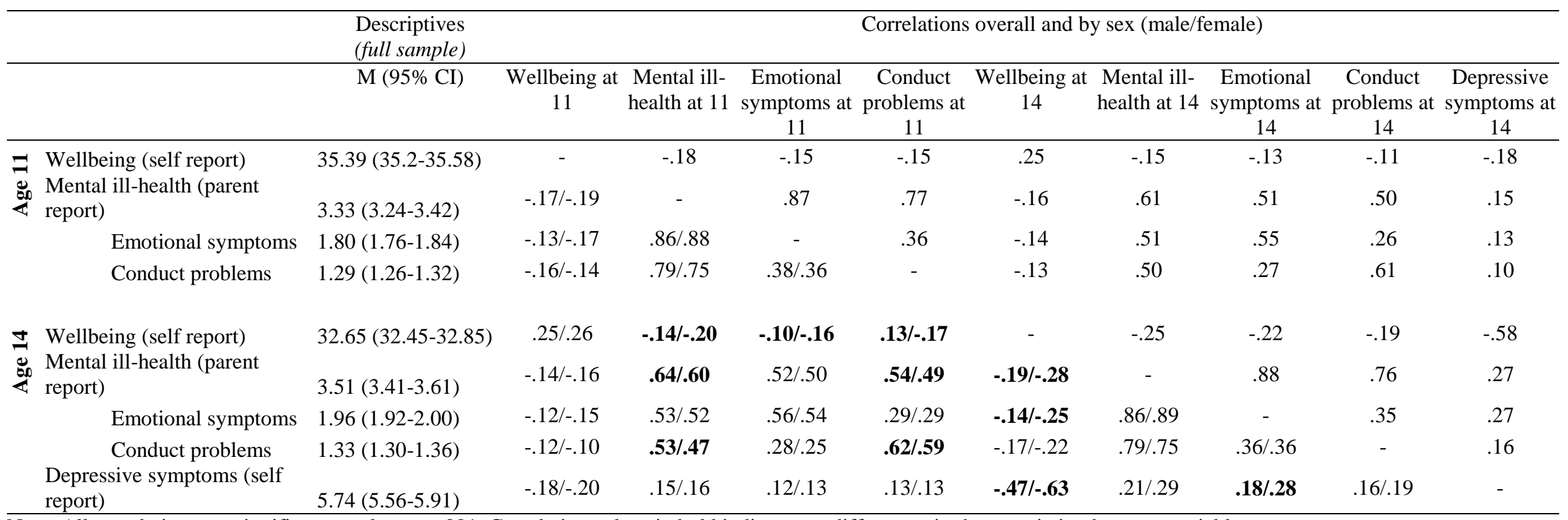

Note: All correlations are significant at atleast $\mathrm{p}<.001$. Correlation values in bold indicate sex differences in the association between variables. 
Table 2 Regression models predicting internalising mental ill-health and mental wellbeing at 14 without and with controlling for mental health at age 11 (coefficients presented are adjusted for all other variables in the models).

\begin{tabular}{|c|c|c|c|c|c|c|}
\hline \multirow{3}{*}{ Predictor } & \multicolumn{3}{|c|}{ Predictors of mental health outcomes at age 14} & \multicolumn{3}{|c|}{$\begin{array}{l}\text { Predictors of mental health outcomes at age } 14 \\
\text { controlling for mental health at age } 11\end{array}$} \\
\hline & $\begin{array}{l}\text { Emotional } \\
\text { symptoms }\end{array}$ & $\begin{array}{l}\text { Depressive } \\
\text { symptoms }\end{array}$ & Wellbeing & $\begin{array}{l}\text { Emotional } \\
\text { symptoms }\end{array}$ & $\begin{array}{l}\text { Depressive } \\
\text { symptoms }\end{array}$ & Wellbeing \\
\hline & Coef $(S E)$ & Coef $(S E)$ & $\operatorname{Coef}(S E)$ & $\operatorname{Coef}(S E)$ & Coef $(S E)$ & Coef $(S E)$ \\
\hline Mental ill-health 11 yrs & & & & $0.41(0.02)^{* * *}$ & $0.04(0.02)^{*}$ & $-0.03(0.02)$ \\
\hline Wellbeing 11 yrs & & & & $-0.03(0.02)$ & $-0.08(0.01)^{* * *}$ & $0.13(0.01)^{* * *}$ \\
\hline Sex (female) & $0.36(0.02)^{* * *}$ & $0.59(0.03)^{* * *}$ & $-0.43(0.03)^{* * *}$ & $0.34(0.02)^{* * *}$ & $0.57(0.03)^{* * *}$ & $-0.41(0.03)^{* * *}$ \\
\hline Ethnicity (Asian) & $-0.05(0.05)$ & $-0.18(0.04) * * *$ & $0.19(0.04)^{* * *}$ & $0.02(0.05)$ & $-0.17(0.04)^{* * *}$ & $0.17(0.04)^{* * *}$ \\
\hline Ethnicity (Black) & $-0.08(0.07)$ & $-0.15(0.08)^{*}$ & $-0.06(0.09)$ & $0.01(0.08)$ & $-0.15(0.08)$ & $-0.06(0.09)$ \\
\hline Ethnicity (Mixed) & $-0.08(0.07)$ & $0.06(0.08)$ & $-0.06(0.07)$ & $-0.10(0.06)$ & $0.07(0.08)$ & $-0.07(0.07)$ \\
\hline Ethnicity (Other) & $0.05(0.13)$ & $-0.04(0.08)$ & $-0.03(0.10)$ & $0.15(0.14)$ & $-0.03(0.08)$ & $-0.05(0.11)$ \\
\hline Income (lowest quintile) & $0.09(0.05)$ & $0.03(0.05)$ & $-0.17(0.06)^{* *}$ & $0.00(0.05)$ & $0.03(0.05)$ & $-0.18(0.06)^{* *}$ \\
\hline Income ( $2^{\text {nd }}$ quintile) & $0.10(0.04)^{*}$ & $0.11(0.04)^{* *}$ & $-0.15(0.04) * * *$ & $0.03(0.04)$ & $0.11(0.04)^{* *}$ & $-0.16(0.04)^{* * *}$ \\
\hline Income ( $3^{\text {rd }}$ quintile) & $0.04(0.04)$ & $0.08(0.04)^{*}$ & $-0.10(0.04)^{*}$ & $0.01(0.03)$ & $0.08(0.04)^{*}$ & $-0.10(0.04)^{*}$ \\
\hline Income ( $4^{\text {th }}$ quintile $)$ & $0.01(0.03)$ & $0.05(0.03)$ & $-0.04(0.04)$ & $-0.00(0.03)$ & $0.05(0.03)$ & $-0.04(0.04)$ \\
\hline Material position of family & $0.01(0.01)$ & $0.05(0.02)^{* * *}$ & $-0.05(0.01)^{* * *}$ & $-0.00(0.01)$ & $0.05(0.02)^{* *}$ & $-0.04(0.01)^{* *}$ \\
\hline Relative wealth (poorer) & $0.00(0.05)$ & $0.08(0.05)$ & $-0.04(0.04)$ & $-0.00(0.04)$ & $0.07(0.05)$ & $-0.03(0.04)$ \\
\hline Relative wealth (richer) & $-0.00(0.07)$ & $0.17(0.07)^{*}$ & $-0.14(0.07)^{*}$ & $0.03(0.07)$ & $0.16(0.07)^{*}$ & $-0.12(0.07)$ \\
\hline Cognitive ability & $-0.09(0.02)^{* * *}$ & $0.07(0.02)^{* * *}$ & $-0.04(0.02)^{*}$ & $-0.05(0.02)^{* * *}$ & $0.08(0.02)^{* * *}$ & $-0.04(0.02)^{*}$ \\
\hline Special educational needs & $-0.04(0.08)$ & $-0.03(0.07)$ & $0.16(0.09)$ & $-0.12(0.08)$ & $-0.03(0.07)$ & $0.16(0.09)$ \\
\hline Communication difficulties & $0.17(0.06)^{* *}$ & $0.04(0.06)$ & $-0.07(0.05)$ & $0.10(0.07)$ & $0.03(0.06)$ & $-0.06(0.05)$ \\
\hline Chronic illness & $0.18(0.04)^{* * *}$ & $-0.01(0.04)$ & $-0.09(0.04)^{*}$ & $0.09(0.04)^{*}$ & $-0.02(0.04)$ & $-0.08(0.04)$ \\
\hline Overweight/obese & $0.02(0.03)$ & $0.12(0.03)^{* * *}$ & $-0.10(0.03)^{* * *}$ & $0.02(0.03)$ & $0.12(0.03)^{* * *}$ & $-0.10(0.03)^{* *}$ \\
\hline Single parent family & $0.04(0.03)$ & $0.06(0.04)$ & $-0.09(0.04)^{*}$ & $-0.01(0.03)$ & $0.05(0.04)$ & $-0.08(0.04)^{*}$ \\
\hline Number of siblings (1) & $0.00(0.04)$ & $-0.04(0.04)$ & $0.05(0.04)$ & $-0.04(0.04)$ & $-0.05(0.04)$ & $0.06(0.04)$ \\
\hline Number of siblings (2) & $-0.03(0.04)$ & $-0.07(0.05)$ & $0.06(0.04)$ & $-0.10(0.04)^{*}$ & $-0.08(0.05)$ & $0.07(0.05)$ \\
\hline Number of siblings (3) & $0.05(0.06)$ & $-0.06(0.06)$ & $0.11(0.05)^{*}$ & $0.00(0.06)$ & $-0.06(0.06)$ & $0.11(0.05)^{*}$ \\
\hline Argue with parent & $0.18(0.03)^{* * *}$ & $0.06(0.03)^{*}$ & $-0.07(0.03)^{* *}$ & $-0.05(0.03)$ & $0.04(0.03)$ & $-0.05(0.03)$ \\
\hline Bullied by siblings & $-0.02(0.03)$ & $0.06(0.03)^{*}$ & $-0.07(0.03)^{* *}$ & $-0.04(0.03)$ & $0.05(0.03)^{*}$ & $-0.06(0.03)^{*}$ \\
\hline Parent mental health & $0.09(0.02)^{* * *}$ & $0.04(0.02)^{*}$ & $0.00(0.02)$ & $0.02(0.02)$ & $0.03(0.02)$ & $0.01(0.02)$ \\
\hline Parent general health & $0.04(0.02)^{*}$ & $0.05(0.02)^{* *}$ & $-0.06(0.01)^{* * *}$ & $0.03(0.02)$ & $0.05(0.02)^{* *}$ & $-0.05(0.01)^{* * *}$ \\
\hline Parent life satisfaction & $-0.04(0.02)^{*}$ & $0.00(0.02)$ & $0.04(0.02)^{*}$ & $-0.04(0.02)^{*}$ & $0.01(0.02)$ & $0.04(0.02)^{*}$ \\
\hline Peer problems & $0.23(0.02)^{* * *}$ & $0.08(0.02)^{* * *}$ & $-0.06(0.01)^{* * *}$ & $0.08(0.02)^{* * *}$ & $0.06(0.02)^{* * *}$ & $-0.03(0.01)^{*}$ \\
\hline Argue with friends & $0.00(0.04)$ & $-0.01(0.04)$ & $-0.05(0.04)$ & $0.00(0.03)$ & $-0.02(0.04)$ & $-0.04(0.04)$ \\
\hline Bullied by peers & $0.06(0.04)$ & $0.21(0.04)^{* * *}$ & $-0.13(0.04)^{* *}$ & $0.03(0.04)$ & $0.20(0.04)^{* * *}$ & $-0.10(0.04)^{*}$ \\
\hline Spends time with friends & $-0.02(0.03)$ & $-0.05(0.03)$ & $0.03(0.03)$ & $-0.02(0.03)$ & $-0.04(0.03)$ & $0.01(0.03)$ \\
\hline School connectedness & $-0.05(0.02)^{*}$ & $-0.14(0.02)^{* * *}$ & $0.15(0.02)^{* * *}$ & $-0.02(0.02)$ & $-0.12(0.02)^{* * *}$ & $0.11(0.02)^{* * *}$ \\
\hline Like school & $0.02(0.03)$ & $0.06(0.03)^{*}$ & $0.12(0.03)^{* * *}$ & $0.04(0.03)$ & $0.08(0.03)^{* *}$ & $0.08(0.03)^{* *}$ \\
\hline Unsafe neighbourhood & $0.10(0.05)^{*}$ & $0.07(0.04)$ & $-0.09(0.04)^{*}$ & $0.10(0.04)^{*}$ & $0.05(0.04)$ & $-0.06(0.04)$ \\
\hline Country (Scotland) & $0.07(0.03)$ & $-0.02(0.03)$ & $0.07(0.05)$ & $0.06(0.03)$ & $-0.02(0.04)$ & $0.07(0.05)$ \\
\hline Country (Wales) & $0.07(0.04)$ & $-0.04(0.04)$ & $0.02(0.04)$ & $0.07(0.04)$ & $-0.03(0.04)$ & $0.01(0.04)$ \\
\hline Country (Northern Ireland) & $0.02(0.04)$ & $-0.08(0.04)^{*}$ & $0.20(0.04)^{* * *}$ & $0.01(0.03)$ & $-0.07(0.04)$ & $0.19(0.04)^{* * *}$ \\
\hline
\end{tabular}

*** $\mathrm{p}<0.001,{ }^{* *} \mathrm{p}<0.01, * \mathrm{p}<0.05$. Note, the first set of models do not control for mental health at age 11 and the second set condition on age 11 mental health. 
Table 3. Regressions predicting internalising symptoms and wellbeing at 14 separately by sex

\begin{tabular}{|c|c|c|c|c|c|c|}
\hline \multirow[b]{2}{*}{ Predictor } & \multicolumn{2}{|c|}{ Emotional symptoms } & \multicolumn{2}{|c|}{ Depressive symptoms } & \multicolumn{2}{|c|}{ Wellbeing } \\
\hline & Males & Females & Males & Females & Males & Females \\
\hline & Coef $(S E)$ & Coef $(S E)$ & Coef (SE) & Coef (SE) & Coef $(S E)$ & Coef $(S E)$ \\
\hline Mental ill-health 11 years & $0.36(0.03)^{* * *}$ & $0.47(0.03)^{* * *}$ & $0.04(0.02)$ & $0.04(0.03)$ & $0.01(0.02)$ & $-0.06(0.02)^{*}$ \\
\hline Wellbeing 11 years & $-0.02(0.02)$ & $-0.03(0.02)$ & $-0.07(0.02)^{* * *}$ & $-0.09(0.02)^{* * *}$ & $0.13(0.02)^{* * *}$ & $0.12(0.02)^{* * *}$ \\
\hline Ethnicity (Asian) & $0.03(0.06)$ & $0.01(0.07)$ & $-0.08(0.05)$ & $-0.22(0.07)^{* *}$ & $0.12(0.05)^{*}$ & $0.21(0.07)^{* *}$ \\
\hline Ethnicity (Black) & $0.19(0.11)$ & $-0.18(0.09)^{*}$ & $-0.00(0.13)$ & $-0.30(0.09)^{* *}$ & $-0.13(0.13)$ & $0.02(0.13)$ \\
\hline Ethnicity (Mixed) & $0.02(0.07)$ & $-0.26(0.09)^{* *}$ & $0.14(0.09)$ & $-0.04(0.13)$ & $-0.03(0.09)$ & $-0.12(0.09)$ \\
\hline Ethnicity (Other) & $0.21(0.20)$ & $0.14(0.11)$ & $0.06(0.12)$ & $-0.12(0.13)$ & $-0.21(0.12)$ & $0.17(0.18)$ \\
\hline Income (lowest quintile) & $-0.00(0.07)$ & $0.01(0.07)$ & $-0.06(0.06)$ & $0.14(0.08)$ & $0.01(0.06)$ & $-0.37(0.09)^{* * *}$ \\
\hline Income ( $2^{\text {nd }}$ quintile $)$ & $0.00(0.05)$ & $0.06(0.06)$ & $0.01(0.05)$ & $0.23(0.07)^{* * *}$ & $-0.06(0.06)$ & $-0.27(0.06)^{* * *}$ \\
\hline Income ( $3^{\text {rd }}$ quintile $)$ & $0.03(0.04)$ & $-0.02(0.05)$ & $0.02(0.05)$ & $0.15(0.06)^{*}$ & $-0.02(0.05)$ & $-0.18(0.06)^{* *}$ \\
\hline Income ( $4^{\text {th }}$ quintile $)$ & $0.02(0.04)$ & $-0.03(0.04)$ & $0.00(0.04)$ & $0.08(0.06)$ & $0.01(0.05)$ & $-0.09(0.05)$ \\
\hline Material position of family & $-0.04(0.02)^{*}$ & $0.04(0.02)$ & $0.02(0.02)$ & $0.06(0.02)^{* *}$ & $-0.04(0.02)^{*}$ & $-0.04(0.02)$ \\
\hline Relative wealth (poorer) & $0.00(0.05)$ & $-0.02(0.08)$ & $0.05(0.04)$ & $0.09(0.10)$ & $-0.01(0.05)$ & $-0.07(0.09)$ \\
\hline Relative wealth (richer) & $-0.10(0.07)$ & $0.17(0.10)$ & $0.11(0.07)$ & $0.22(0.11)^{*}$ & $-0.05(0.09)$ & $-0.21(0.09)^{*}$ \\
\hline Cognitive ability & $-0.08(0.02)^{* * *}$ & $-0.03(0.02)$ & $0.05(0.02)^{* *}$ & $0.12(0.03)^{* * *}$ & $-0.03(0.02)$ & $-0.06(0.02)^{* *}$ \\
\hline Special educational needs & $-0.10(0.09)$ & $-0.14(0.15)$ & $0.06(0.08)$ & $-0.26(0.13)$ & $0.05(0.10)$ & $0.38(0.14)^{* *}$ \\
\hline Communication difficulties & $0.08(0.07)$ & $0.16(0.14)$ & $0.08(0.08)$ & $-0.03(0.09)$ & $-0.05(0.07)$ & $-0.08(0.11)$ \\
\hline Chronic illness & $0.13(0.05)^{* *}$ & $0.05(0.05)$ & $-0.05(0.04)$ & $0.01(0.07)$ & $-0.09(0.06)$ & $-0.08(0.06)$ \\
\hline Overweight/obese & $-0.00(0.04)$ & $0.05(0.04)$ & $0.08(0.04)^{*}$ & $0.15(0.04)^{* * *}$ & $-0.06(0.04)$ & $-0.13(0.04)^{* *}$ \\
\hline Single parent family & $-0.01(0.04)$ & $-0.02(0.05)$ & $-0.01(0.04)$ & $0.10(0.06)$ & $-0.06(0.05)$ & $-0.09(0.05)$ \\
\hline Number of siblings (1) & $0.02(0.05)$ & $-0.09(0.06)$ & $-0.00(0.05)$ & $-0.07(0.07)$ & $0.01(0.06)$ & $0.09(0.06)$ \\
\hline Number of siblings (2) & $-0.06(0.05)$ & $-0.12(0.06)$ & $-0.02(0.06)$ & $-0.12(0.08)$ & $-0.02(0.06)$ & $0.14(0.07)$ \\
\hline Number of siblings (3) & $0.08(0.07)$ & $-0.05(0.08)$ & $-0.01(0.07)$ & $-0.11(0.09)$ & $0.02(0.07)$ & $0.19(0.07)^{*}$ \\
\hline Argue with parent & $-0.05(0.04)$ & $-0.05(0.04)$ & $0.01(0.04)$ & $0.08(0.04)$ & $-0.07(0.04)$ & $-0.05(0.04)$ \\
\hline Bullied by siblings & $-0.03(0.04)$ & $-0.06(0.04)$ & $0.10(0.03)^{* *}$ & $-0.01(0.04)$ & $-0.05(0.03)$ & $-0.06(0.04)$ \\
\hline Parent mental health & $0.04(0.03)$ & $0.01(0.03)$ & $0.01(0.02)$ & $0.06(0.03)$ & $0.01(0.02)$ & $0.00(0.03)$ \\
\hline Parent general health & $0.02(0.02)$ & $0.04(0.02)$ & $0.05(0.02)^{*}$ & $0.04(0.02)$ & $-0.06(0.02)^{* *}$ & $-0.04(0.02)^{*}$ \\
\hline Parent life satisfaction & $-0.02(0.03)$ & $-0.05(0.02)^{*}$ & $-0.01(0.02)$ & $0.03(0.02)$ & $0.05(0.03)^{*}$ & $0.02(0.02)$ \\
\hline Peer problems & $0.08(0.02)^{* *}$ & $0.08(0.02)^{* * *}$ & $0.05(0.02)^{*}$ & $0.07(0.03)^{*}$ & $-0.05(0.02)^{*}$ & $-0.02(0.02)$ \\
\hline Argue with friends & $0.07(0.05)$ & $-0.06(0.05)$ & $-0.01(0.05)$ & $-0.05(0.06)$ & $-0.04(0.05)$ & $-0.01(0.06)$ \\
\hline Bullied by peers & $0.08(0.05)$ & $-0.04(0.06)$ & $0.17(0.05)^{* * *}$ & $0.23(0.07)^{* * *}$ & $-0.11(0.05)^{*}$ & $-0.09(0.06)$ \\
\hline Spends time with friends & $-0.07(0.04)$ & $0.03(0.04)$ & $-0.07(0.03)^{*}$ & $-0.01(0.04)$ & $0.03(0.03)$ & $-0.02(0.04)$ \\
\hline School connectedness & $-0.04(0.02)$ & $-0.00(0.03)$ & $-0.08(0.02)^{* * *}$ & $-0.19(0.03)^{* * *}$ & $0.07(0.02)^{* * *}$ & $0.18(0.03)^{* * *}$ \\
\hline Like school & $0.10(0.03)^{* *}$ & $0.00(0.04)$ & $0.11(0.03)^{* *}$ & $0.07(0.05)$ & $0.08(0.04)^{*}$ & $0.06(0.05)$ \\
\hline Unsafe neighbourhood & $0.05(0.06)$ & $0.15(0.07)^{*}$ & $0.03(0.06)$ & $0.09(0.07)$ & $-0.05(0.06)$ & $-0.07(0.06)$ \\
\hline Country (Scotland) & $0.07(0.04)$ & $0.05(0.05)$ & $0.02(0.04)$ & $-0.04(0.06)$ & $0.02(0.06)$ & $0.11(0.06)$ \\
\hline Country (Wales) & $0.02(0.05)$ & $0.12(0.05)^{*}$ & $0.01(0.04)$ & $-0.07(0.05)$ & $0.00(0.05)$ & $0.02(0.05)$ \\
\hline Country (Northern Ireland) & $0.03(0.05)$ & $-0.01(0.05)$ & $-0.00(0.05)$ & $-0.15(0.06)^{*}$ & $0.10(0.05)$ & $0.27(0.05)^{* * *}$ \\
\hline
\end{tabular}

*** $\mathrm{p}<0.001, * * \mathrm{p}<0.01,{ }^{*} \mathrm{p}<0.05$. Note: Coefficients in bold are those that were significantly different by sex based on sex*predictor interactions 